\title{
On asymptotic optimality of Merton's myopic portfolio strategies under time discretization
}

\author{
Alexandra Rodkina *and Nikolai Dokuchaev ${ }^{\ddagger}$ \\ Submitted: March 18, 2014. Revised: November 24, 2014. Accepted: April 13, 2015
}

\begin{abstract}
This paper studies the properties of discrete time stochastic optimal control problems associated with portfolio selection. We investigate if optimal continuous time strategies can be used effectively for a discrete time market after a straightforward discretization. We found that Merton's strategy approximates the performance of the optimal strategy in a discrete time model with the sufficiently small time steps.
\end{abstract}

Keywords: optimal portfolio, utility, discretization, discrete time Itô formula MSC: 93E20, 91G10.

\section{Introduction}

The paper studies discrete time stochastic optimal control problems and their relationships with continuous time optimal control problems. More precisely, we study optimal investment problems where $\mathbf{E} U\left(X_{T}\right)$ is to be maximized. Here $X_{T}$ represents the total wealth at final time $T$, and $U(\cdot)$ is a utility function. We consider the case where $U(x)=x^{\alpha}, \alpha \in(0,1)$. For continuous time market models, these utilities have a special significance, in particular, because the optimal strategies for them are known explicitly (so-called Merton's strategies). These strategies are myopic; they depend only on the current observations of the market parameters, including the risk free rate, the appreciation rate, and the volatility matrix, even for the case of unknown prior distributions and evolution law. The optimality of Merton's strategies still

\footnotetext{
${ }^{*}$ Department of Mathematics, The University of the West Indies, Kingston 7, Jamaica

${ }^{\dagger}$ Department of Mathematics and Statistics, Curtin University, Perth, WA, Australia

${ }^{\ddagger}$ Corresponding author

This is a pre-copy-editing, author-produced PDF of an article accepted in IMA Journal Of Mathematical Control And Information following peer review. The definitive publisher-authenticated version will be available online at http://imamci.oxfordjournals.org/
} 
holds when the market parameters are random and independent of the driving Brownian motion, i.e., in the case of "totally unhedgeable" coefficients, according to Karatzas and Shreve (1998), Chapter 6. The solution that leads to myopic strategies goes back to Merton (1969); the case of random coefficients was discussed in Karatzas and Shreve (1998) and Dokuchaev and Haussmann (2001).

The real stock prices are presented as time series, so the discrete time (multi-period) models are more natural than continuous time models. On the other hand, continuous-time models give a good description of distributions and often allow explicit solutions of optimal investment problems.

For a real market, a formula for an optimal strategy derived for a continuous-time model can often be effectively used after the natural discretization. However, this strategy will not be optimal for time series observed in the real market. Therefore, it is important to extend the class of discrete time models that allow myopic and explicit optimal portfolio strategies. The problem of discrete-time portfolio selection has been studied in the literature, such as in Smith (1967); Leland (1968); Mossin (1968); Merton (1969); Samuelson (1969); Fama (1970); Hakansson (1971a); Hakansson (1971b); Elton and Gruber (1974); Francis (1976); Dumas and Liucinao (1991); Östermark (1901); Grauer and Hakansson (1993); Pliska (1997); Li and Ng (2000); Xu et al (2008); Çanakoğlu and Özekici (2009); Zhang and Li (2012). If a discrete time market model is complete, then the martingale method can be used (see, e.g., Pliska (1997)). Unfortunately, a discrete time market model can be complete only under very restrictive assumptions. For incomplete discrete time markets, the main tool is dynamic programming that requires to derive and solve a backward Bellman equation with a Cauchy condition at the terminal time. For the general case, this procedure involves recalculation of the conditional densities at each time step which is numerically challenging (see, e.g., Pliska (1997) or Gikhman and Skorohod (1979)). This is why the optimal investment problems for discrete time can be more difficult than for the continuous time setting where explicit solutions are often possible.

There are several special cases when an investment problem allows for an explicit solution in discrete time, and, for some cases, optimal strategies are myopic; see Leland (1968); Mossin (1968); Hakansson (1971a); Dokuchaev (2007a, 2010a)). However, the optimal strategy is not myopic and it cannot be presented explicitly for power utilities in the general case. Hakansson (1971a) showed that the optimal strategy is not myopic for $U(x)=\sqrt{x}$ if returns have serial correlation and evolve as a Markov process.

In a mean-variance discrete time multi-period setting, the optimal strategies represent some analog of Merton's strategies. These strategies are myopic for mean-variance goal achieving 
problems and non-myopic if these goals have to be selected to solve a problem with constraints; see Li and Ng (2000), Dokuchaev (2010b), Zhang and Li (2012). It appears that the problems with utility functions $U(x)=x^{\alpha}, \alpha<1$, have different properties with respect to time disretization. In particular, Dokuchaev (2007a) demonstrated that the direct discretization of continuous time optimal Merton's strategies does not approximate the optimal strategy for the discrete time market for concave utility functions $U(x)=x^{\alpha}$ such that $U(x)=-\infty$ for $x<0$. More precisely, the difference between the optimal expected utilities for discrete time and continuous time models does not disappear if the number of periods (or frequency of adjustments) grows. As the result, the optimal expected utility calculated for a continuous time market cannot be approximated by piecewise constant strategies with possible jumps at given times $\left(t_{k}\right)_{k=1}^{N}$, even if $N \rightarrow+\infty$ and $t_{k}-t_{k+1} \rightarrow 0$.

In the present paper, we reconsider the discrete time optimal portfolio selection problems. We suggest a solution based on myopic Merton's strategies that are optimal for related continuous time portfolio selection problems. We investigate the limit properties of the discrete time optimal portfolio selection problem when the step of the discretization converges to zero. We found that the performance of the discrete time strategy obtained directly from Merton's strategy approximates the optimal strategy. This suboptimal discrete time strategy is myopic. We consider the case of Gaussian noise in the discrete time equation for the price. This means that the stock price can be negative with non-zero probability; this feature does not affect the validity of the model since this probability converges to zero as the step of discretization converges to zero; see Appleby et al (2010). The proof is based on the application of the variant of the discrete Itô formula first introduced by Appleby, Berkolaiko and Rodkina (2009). It can be noted that the proof does not use the dynamic programming principle.

These results lead to a conclusion that Merton's strategies can be used effectively for the discrete time multi-period market models with power utilities $U(x)=x^{\alpha}, \alpha<1$ that have sufficiently small time steps and approximate the continuous time model. This seems to contradict to the result from Dokuchaev (2007a). However, there is not a contradiction. In the present paper, we assumed that $U(x)=L x$ for $x<0$, where $L>0$ can be selected to be arbitrarily large. On the other hand, Dokuchaev (2007a) assumed that $U(x)=-\infty$ for $x<0$. This difference in the problem setting leads to different conclusions. Note that the utility function considered in the present paper is not concave; however, its shape is becoming "more concave" as $L \rightarrow+\infty$. Moreover, the impact of non-concavity of $U$ for any given $L$ disappears since this probability converges to zero as the step of discretization converges to zero. We illustrate this in numerical experiments to demonstrate the impact of the size of the interval of discretization on the performance of Merton's strategy and the impact of the selection of finite $L$ in the adjusted 
utility function. In these experiments, we found that weekly portfolio adjustments is sufficient to compensate the discretization error for Merton's strategy. Moreover, we found that this error is almost negligible for the model with daily portfolio adjustments.

\section{$2 \quad$ Problem setting}

In this paper we consider the following controlled stochastic difference equation

$$
\begin{aligned}
& x_{n+1}=x_{n}\left(1+h u_{n} a_{n}+\sqrt{h} u_{n} b_{n} \xi_{n+1}\right), \quad n=0,1, \ldots, N-1, \\
& x(0)=x_{0}>0
\end{aligned}
$$

where $x_{0}>0$ is nonrandom, $\xi_{n}$ are random variables, $a_{n}, b_{n}$ are nonrandom coefficients, $u_{n}$ is a nonrandom control (strategy), $n=0,1, \ldots, N-1, N \in \mathbf{N}, h>0$ is a small parameter, calculated as

$$
h:=\frac{T}{N}
$$

The value $T>0$ is fixed throughout all paper, but $N$ can increase, which makes $h$ decrease.

We can either consider equation (2.1) independently, or think about it as the Eulier-Maruyama discretization of the following Itô stochastic equation

$$
d X_{t}=X_{t} u(t)\left(a(t) d t+b(t) d W_{t}\right), \quad t \in[0, T], \quad X(0)=x_{0},
$$

where $W$ is a standard Wiener process, $b, a, u:[0, T] \rightarrow \mathbf{R}$ are continuous nonrandom functions. In this setting $h$ is a step size of discretization of the interval $[0, T]$ and $N$ is a number of corresponding mesh points.

We recall that the Euler-Maruyama numerical method for equation (2.3) computes approximations $x_{n}(h) \approx X_{n h}$ by

$$
x_{n+1}(h)=x_{n}(h)\left(1+h u(n h) a(n h)+u(n h) b(n h) \Delta W_{n+1}\right),
$$

where $h>0$ is the constant step size and $\Delta W_{n+1}=W((n+1) h)-W(n h)$. We see that when

$$
\xi_{n+1}=\frac{W((n+1) h)-W(n h)}{\sqrt{h}}, \quad a_{n}=a(n h), \quad b_{n}=b(n h), \quad u_{n}=u(n h),
$$

(2.4) coincides with (2.1) and $\xi_{n+1}$ is a standardized normal random variable. More information about Euler-Maruyama discretization and stochastic difference equations could be found, e.g., in Higham et al (2002); Kloeden and Platen (1992); Appleby et al (2010); Appleby, Berkolaiko and Rodkina (2009).

Assume that the following assumptions hold. 
Assumption 2.1. Sequences $\left(a_{n}\right)_{n \in \mathbf{N}}$ and $\left(b_{n}\right)_{n \in \mathbf{N}}$ are nonrandom and such that for some $\hat{a}, \hat{b}, \underline{a}, \underline{b}>0, \quad \underline{a} \leq \hat{a}, \quad \underline{b} \leq \hat{b}$,

$$
\underline{a} \leq\left|a_{n}\right| \leq \hat{a}, \quad \underline{b} \leq\left|b_{n}\right| \leq \hat{b}, \quad \forall n \in \mathbf{N} .
$$

Assumption 2.2. $\left(\xi_{n}\right)_{n \in \mathbf{N}}$ is a sequence of independent and $\mathcal{N}(0,1)$ distributed random variables.

Let $\left(\Omega, \mathcal{F},\left\{\mathcal{F}_{n}\right\}_{n \in \mathbf{N}}, \mathbb{P}\right)$ be a complete filtered probability space. We assume that the filtration $\left\{\mathcal{F}_{n}\right\}_{n \in \mathbf{N}}$ is naturally generated: $\mathcal{F}_{n+1}=\sigma\left\{\xi_{i+1}: i=0,1, \ldots, n\right\}$, where the sequence $\left(\xi_{n}\right)_{n \in \mathbf{N}}$ satisfies Assumption 2.2.

We use the standard abbreviation "a.s." for the wordings "almost sure" or "almost surely" throughout the text.

Among all the sequences $\left(x_{n}\right)_{n \in \mathbf{N}}$ of the random variables we distinguish those for which $x_{n}$ are $\mathcal{F}_{n}$-measurable for all $n \in \mathbf{N}$. A detailed exposition of the definitions and facts of the theory of random processes can be found, e.g., in Shiryaev (1996).

Define for some $\alpha \in(0,1)$ and $L>0$,

$$
U(x)=x^{\alpha}, x \geq 0, \quad U(x)=L x, x<0 .
$$

Definition 2.1. For a given $N \in \mathbf{N}$, the set $\mathcal{U}=\mathcal{U}(N)$ of admissible strategies is the set of all nonrandom vectors $u=\left(u_{n}\right)_{n=0}^{N-1}$ such that

$$
\underline{u} \leq\left|u_{n}\right| \leq \hat{u}, \quad n=0,1, \ldots, N-1,
$$

for some positive numbers $\underline{u}, \hat{u}, \quad 0<\underline{u} \leq \hat{u}$.

Up to the end of the paper, we consider the following optimal control problem:

$$
\text { Maximize } \mathbf{E}\left[U\left(x_{N}\right)\right] \text { over } u \in \mathcal{U}
$$

where $x$ is a solution to (2.1) with $h=\frac{T}{N}$ and admissible strategy $u, \mathcal{U}=\mathcal{U}(N)$ is the set of all admissible strategies introduced in Definition 2.1.

\section{Optimal portfolio selection and the main result}

Problem (2.8) has applications for optimal portfolio selection. It appears that (2.1) describes the dynamic of the total wealth $x_{n}$ of an investor at time period $n$ for a single stock discrete time market model with a risk-free investment with zero return. The dynamic of the stock price is described by the equation

$$
s_{n+1}=s_{n}\left(1+h a_{n}+\sqrt{h} b_{n} \xi_{n+1}\right), \quad n=0,1, \ldots, N-1, \quad s_{0}=1 .
$$


It is assumed that the portfolio is distributed among the shares of this stock and the risk-free investment with zero return. A strategy $u$ represents a dynamically selected ratio of investment in stock. More precisely, let $\gamma_{n}$ be the quantity of stock shares in the portfolio at time $n$, then $u_{n}=\gamma_{n} s_{n} / x_{n}$, where $\gamma_{n} s_{n}$ is the current value of the stock portfolio, $x_{n}$ is the current total value of the portfolio. We select the strategy $u$ in the class of admissible processes described above and calculate the quantity of shares $\gamma_{n}=u_{n} x_{n} / s_{n}$; effectively, we select closed-loop strategies. It is assumed that the strategy is self-financing, i.e.,

$$
x_{n+1}-x_{n}=\gamma_{n}\left(s_{n+1}-s_{n}\right), \quad n=0,1,2, \ldots
$$

where $\gamma_{n}=u_{n} x_{n} / s_{n}$ is the quantity of stock shares in the portfolio at time $n$. This assumptions means that the model does not include an external sources of funds and that there is no expenses, transaction costs, and dividend payments. The increments of the wealth are defined solely by the stock price changes and by the quantity of the shares.

In fact, the case of non-zero return for the risk free asset is also covered by this model, if one interprets $x_{n}$ as the discounted wealth and $s_{n}$ as the discounted stock price (discounted with respect to the risk-free asset). A more detailed market model description can be found, e.g., in Pliska (1997); Dokuchaev (2007a).

For this discrete time market model, a standard problem of optimal portfolio selection is to maximize the expectation of the utility function $\mathbf{U}\left(x_{N}\right)$ of the terminal wealth $x_{N}$, i.e., to find a strategy $u^{*}$ which solves optimal control problem

$$
\text { Maximize } \mathbf{E}\left[\mathbf{U}\left(x_{N}\right)\right] \text { over } \mathcal{U}
$$

where $\mathbf{U}$ is some given concave utility function, $x$ is a solution to $(2.1)$ with $h=\frac{T}{N}, \mathcal{U}$ is a set of all admissible strategies according to Definition 2.1.

Further, Itô equation (2.3) describes the evolution of the total wealth $X_{t}$ for a single stock continuous market model with zero risk-free interest rate where the stock price evolution is described by the Itô equation

$$
d S_{t}=S_{t}\left(a(t) d t+b(t) d W_{t}\right), \quad t \in[0, T], \quad S_{0}=1 .
$$

For this continuous time market model, a standard optimal portfolio selection problem is to maximize the expectation of the utility function $\mathbf{U}\left(X_{T}\right)$ of the terminal wealth $X_{T}$, i.e., to find a strategy $u:[0, T] \times \Omega \rightarrow \mathbf{R}$ in a certain class of admissible strategies $\overline{\mathcal{U}}$ that solves optimal control problem

$$
\text { Maximize } \mathbf{E}\left[\mathbf{U}\left(X_{T}\right)\right] \text { over } \overline{\mathcal{U}}
$$


where $X_{t}$ is a solution to (2.3). For the case when $\mathbf{U}(x)=x^{\alpha}, \alpha \in(0,1)$, the following so-called Merton strategy

$$
u^{*}(t)=\frac{a(t)}{(1-\alpha) b^{2}(t)}, \quad t \in[0, T],
$$

is optimal in the continuous time setting (3.4) in the class of admissible strategies that include all bounded random processes adapted to the filtration generated by $S_{t}$; see, e.g., Karatzas and Shreve (1998), Chapter 6, and Merton (1969). In fact, this strategy is optimal in a even wider class of random and adapted processes $u(t)$, as well as in the setting with random $a(t)$ and $b(t)$ that are independent from $W_{t}$.

It can be seen that problem (2.8) is in fact a modification of problem (3.2). Note that the "utility function" $U(x)$ in (2.8) is not concave in $x \in R$; however, its shape is becoming "more concave" as $L \rightarrow+\infty$.

Consider the strategy $u^{*}$ such that

$$
u_{n}^{*}=\frac{a_{n}}{(1-\alpha) b_{n}^{2}}, \quad n=0,1, \ldots, N-1 .
$$

Condition (2.7) is satisfied for this strategy. Notice that this strategy represents a direct discretization of Merton's strategy (3.5). It can be also noted that strategy (3.6) does not depend on the choice of $L$.

Our main result can be formulated as the following.

Theorem 3.1. The strategy $u^{*}$ defined by (3.6) maximizes $\mathbf{E} U\left(x_{N}\right)$ approximately for small enough $h=\frac{T}{N}$, meaning that

$$
\sup _{u} \mathbf{E} U\left(x_{N}\right)=\mathbf{E} U\left(x_{N}^{*}\right)+O(h) \quad \text { as } \quad h \rightarrow 0,
$$

where $x_{N}^{*}$ is the terminal wealth for strategy $(3.6)$ and $O(h) \rightarrow 0$ as $h \rightarrow 0$, independently on $N$.

We show that the error of this approximation tends to zero as step size of discretization $h \rightarrow 0$ (which is equivalent that number of mesh points $N \rightarrow \infty$ ). The proof is heavily dependent on the application of the variant of the discrete Itô formula first introduced in Appleby, Berkolaiko and Rodkina (2009), as well as on the fact that solution $x_{n}^{*}$ of (2.1) for strategy (3.6) is positive for all $n=1, \ldots, N$ with probability which tends to one when $h \rightarrow 0$ (or $N \rightarrow \infty$ ); see Appleby et al (2010).

In Dokuchaev (2007a), it was shown that the direct discretization of continuous time optimal Merton's strategies does not approximate the optimal strategy for the discrete time market if the utility function $U(x)=x^{\alpha}$ is extended as $U(x)=-\infty$ for $x<0$. We found that this can be overcome using the functions $U$ with non-concavity that can be made arbitrarily small with selection of a large $L>0$. Moreover, we show that the probability that this non-concavity will 
ever have any impact vanishes as $h \rightarrow 0$, since the probability that the wealth ever achieves zero vanishes as $h \rightarrow 0$.

Let us review the main steps of the proofs.

Let

$$
\phi(x)=|x|^{\alpha}, \quad x \in \mathbf{R} .
$$

First, we observe that the solution $x_{n}$ of (2.1) can be represented as

$$
x_{n}=x_{0} \prod_{i=0}^{n-1}\left(1+h u_{i} a_{i}+\sqrt{h} u_{i} b_{i} \xi_{i+1}\right), \quad x_{0}>0, \quad n=1, \ldots, N .
$$

Hence

$$
\mathbf{E} \phi\left(x_{n}\right)=\phi\left(x_{0}\right) \prod_{i=0}^{n-1} \mathbf{E} \phi\left(1+h u_{i} a_{i}+\sqrt{h} u_{i} b_{i} \xi_{i+1}\right), \quad n=1, \ldots, N
$$

Application of the discrete Itô formula to each $\mathbf{E} \phi\left(1+h u_{i} a_{i}+\sqrt{h} u_{i} b_{i} \xi_{i+1}\right)$ gives that

$$
\sup _{u} \mathbf{E} \phi\left(x_{N}\right)=x_{0}^{\alpha} \prod_{n=0}^{N-1}\left[1+\alpha h \frac{a_{n}^{2}}{2(1-\alpha) b_{n}^{2}}\right]+O(h) \quad \text { as } \quad h \rightarrow 0 .
$$

Then we show that the probability

$$
\mathbf{P}\left\{\omega: U\left(x_{N}(\omega)\right) \neq \phi\left(x_{N}(\omega)\right)\right\}
$$

can be made arbitrary small when $N=T / h$ is big enough. Finally we prove that

$$
\sup _{u} \mathbf{E}\left[U\left(x_{N}\right)\right]=x_{0}^{\alpha} \prod_{n=0}^{N-1}\left[1+\alpha h \frac{a_{n}^{2}}{2(1-\alpha) b_{n}^{2}}\right]+O(h) \quad \text { as } \quad h \rightarrow 0 .
$$

\section{Simulations}

The equation of type (2.1) with $u_{n} \equiv 1, a_{n} \equiv \lambda, b_{n} \equiv \mu$ was considered in Palmer (2012), where an explicit bound on $h$ suitable for application of the discrete Itô formula was computed and error terms were estimated.

Appleby et al (2010) developed an asymptotic estimate on the number of mesh points $N(\gamma)$ required to ensure the positivity of solutions of Euler-Maruyama discretization of (2.3) as the required proportion of positive trajectories $\gamma$ approaches 1 . Based on the above works one can estimate $h$ (or $N$ ) for equation (2.1) in order to be able to apply the discrete Itô formula and to ensure positivity with given probability $\gamma$.

For the purposes of the present paper, it suffices to demonstrate the impact of the sampling interval of discretization on the performance of Merton's strategy. In addition, we want to show the impact of the selection of finite $L$ in the adjusted utility function $U=U_{L}$ defined by (2.6). 


\begin{tabular}{|c|c|c|c|c|c|}
\hline $\mathrm{L}$ & 2 & 6 & 12 & 52 & 250 \\
\hline 10 & 0.054938 & 0.004632 & 0.001674 & 0.000803 & $9.506101 \times 10^{-5}$ \\
\hline $10^{2}$ & 0.462369 & 0.010440 & 0.001706 & 0.000803 & $9.506101 \times 10^{-5}$ \\
\hline $10^{3}$ & 4.536681 & 0.068490 & 0.002021 & 0.000803 & $9.506101 \times 10^{-5}$ \\
\hline $10^{5}$ & 452.7109 & 6.453968 & 0.036671 & 0.000803 & $9.506101 \times 10^{-5}$ \\
\hline $10^{6}$ & 4527.022 & 64.50377 & 0.351671 & 0.000803 & $9.506101 \times 10^{-5}$ \\
\hline
\end{tabular}

Table 1: The differences $\sup _{N, u} E X_{T}^{\alpha}-E U\left(X_{N}\right)$ in Theorem 3.1 for different values of $L$ in (2.6) and for different numbers $N$ of portfolio adjustments during one year time period.

We remind that the classical concave utility function corresponds to the case $L=+\infty$ that we excluded.

Table 1 presents the results of the numerical simulations with Matlab. This table shows the differences $E U\left(X_{T}^{*}\right)-E U\left(x_{N}^{*}\right)$, where $E U\left(x_{N}^{*}\right)$ is the expected utility for the strategy described in Theorem 3.1, and where

$$
E U\left(X_{T}^{*}\right)=\exp \left(\frac{a}{2 b^{2}} \frac{\alpha}{1-\alpha}\right)
$$

is the expected utility for Merton's strategy in the continuous time setting. Note that the value $X_{T}^{*}$ is non-negative; therefore $E U\left(X_{T}^{*}\right)=E X_{T}^{* \alpha}$, and this value is not impacted by the choice of $L$. The table shows the values of these differences for the parameters $L=10,10^{2}, 10^{3}, 10^{5}, 10^{6}$ presented in (2.6) and for $N=2,6,12,52,250$, with $X_{0}=1, T=1, \alpha=1 / 2, T=1, a=0.07$, $b=0.2$, with 1,000,000 simulations each. For these parameters, $E U\left(X_{T}^{*}\right)=1.0631$.

The values $N$ show the numbers of allowed portfolio adjustments during one year time period, with $\delta=T / N=1 / N$.

It can be seen that $n=52$ (i.e., $\delta=1 / 52$ ) that corresponds to weekly portfolio adjustments is sufficient to compensate the discretization error for Merton's strategy. This error is almost negligible for $n=250$ (i.e., $\delta=1 / 250$ ) that corresponds to daily portfolio adjustments.

\section{Conclusions}

We have investigated the possibility of using known optimal continuous time strategies for solving the discrete time optimal portfolio selection problems. For this, we studied the limit properties of the discrete time optimal portfolio selection problem when the step of the discretization converges to zero. We found that the performance of the discrete time strategy obtained directly 
from Merton's strategy approximates the optimal strategy after some minor adjustment of the utility function. This suboptimal discrete time strategy is myopic. The proof is based on the application of a discrete Itô formula. The results of this paper leads to the conclusion that Merton's strategies can be used effectively for discrete time multi-period market models.

\section{Acknowledgment}

The authors thank Chuong Luong for the help with Matlab calculations. In addition, the authors thank the anonymous referees for their valuable remarks which helped us to improve the paper.

\section{Funding}

This work was supported by ARC grant of Australia DP120100928.

\section{References}

Appleby, J. A. D., Berkolaiko, G., and Rodkina, A. (2009). Non-exponential stability and decay rates in nonlinear stochastic difference equation with unbounded noises. Stochastics: An International Journal of Probability and Stochastic Processes, 81 (2), 99-127.

Appleby, J. A. D., Guzowska, M., Rodkina, A., and Kelly C. (2010). Preservation of positivity in the solution of discretised stochastic differential equation, Applied Mathematics and Computation 217, (2010), 763-774.

Berkolaiko, G., Buckwar, E., Kelly, C., and Rodkina, A. (2012) Almost sure asymptotic stability analysis of the $\theta$-Maruyama method applied to a test system with a.s. stabilising and destabilising stochastic perturbations. LMS Journal of Computation and Mathematics, 15:71-83.

Çanakoğlu, E., and Özekici, S (2010). Portfolio selection in stochastic markets with HARA utility functions. European Journal of Operational Research 201, 520-536.

Dokuchaev, N.G. (2002). Dynamic portfolio strategies: quantitative methods and empirical rules for incomplete information. Kluwer, Boston.

Dokuchaev, N. (2007a). Discrete time market with serial correlations and optimal myopic strategies. European Journal of Operational Research 177, iss. 2, 1090-1104. 
Dokuchaev, N. (2007b). Mathematical finance: core theory, problems, and statistical algorithms. Routledge, London and New York.

Dokuchaev, N. (2010a). Optimality of myopic strategies for multi-stock discrete time market with management costs. European Journal of Operational Research 200, Issue 2, 551-556.

Dokuchaev, N. (2010b). Mean variance and goal achieving portfolio for discrete-time market with currently observable source of correlations. ESAIM: Control, Optimization and Calculus of Variations 16, Number 3, 635-647.

Dokuchaev, N.G. and Haussmann, U. (2001). Optimal portfolio selection and compression in an incomplete market. Quantitative Finance 1 (3), 336-345.

Dumas, B., and Liucinao, E. (1991). An exact solution to a dynamic portfolio choice problem under transaction costs, Journal of Finance 46, 577-595.

Elton, E. J., and Gruber, M.J. (1974). On the optimality of some multiperiod portfolio selection criteria, Journal of Business, 231-243.

Fama, E. F. (1970). Multi-period consumption-investment decisions, American Economic Review 60, 163-174.

Francis, J. C. (1976). Investments : Analysis and Management. New York: McGraw-Hill.

Gikhman, I.I., and Skorohod, A.V. (1979). Controlled stochastic processes, Springer-Verlag, New York-Heidelberg.

Grauer, R. R., and Hakansson, N.H. (1993). On the use of mean variance and quadratic approximations in implementing dynamic investment strategies: a comparison of returns and investment policies, Management Science 39, 856-871.

Hakansson, N. H. (1971a). On optimal myopic portfolio policies, with and without serial correlation of yields, Journal of Business 44, 324- 334.

Hakansson, N.H. (1971b). Multi-period mean-variance analysis : Toward a general theory of portfolio choice, Journal of Finance 26, 857-884.

Higham, D,J., Mao, X., and Stuart, A.M. (2002). Strong convergence of numerical methods for nonlinear stochastic differential equations, SIAM J. Num. Anal. 40 (3), 1041-1063.

Karatzas, I., and Shreve, S.E. (1991). Brownian Motion and Stochastic Calculus, Springer, New York. 
Karatzas, I., and Shreve, S.E. (1998). Methods of Mathematical Finance. New York: SpringerVerlag.

Kloeden, P.E., and Platen, E. (1992). Numerical Solution of Stochastic Differential Equations, Springer-Verlag, Berlin.

Leland, H. (1968). Dynamic Portfolio Theory. PhD Thesis, Harvard University.

Li, Duan, and Ng, Wan Lung. (2000). Optimal portfolio selection: multi-period mean-variance optimization. Mathematical Finance 10 (3), 387-406.

Merton, R. (1969). Lifetime portfolio selection under uncertainty: the continuous-time case. Review of Economics and Statistics 51, 247-257.

Mossin, J. (1968). Optimal multi-period portfolio policies, Journal of Business 41, 215-229.

Östermark, R. (1991). Vector forecasting and dynamic portfolio selection: Empirical efficiency of recursive multi-period strategies, European Journal of Operational Research 55, 46-56.

Palmer, P.C. (2012) Application of a discrete Itô formula to determine stability (instability) of the equilibrium a scalar linear stochastic difference equation. Computers and Mathematics with Applications, 64 , 2302-2311.

Pliska, S. R. (1997). Introduction to mathematical finance: discrete time models. Blackwell Publishers.

Rodkina, A., and Dokuchaev, N. (2013). Instability and stability of solutions of systems of nonlinear stochastic difference equations with diagonal noise, Journal of Difference Equations and Applications, 2014, 20.,5-6, 744-764.

Samuelson, P. A. (1969). Lifetime portfolio selection by dynamic stochastic programming, The Review of Economics and Statistics 50, 239-246.

Smith, K. V. (1967). A transition model for portfolio revision, Journal of Finance 22, 425-439.

Shiryaev, A.N. (1996). Probability (2nd edition), Springer, Berlin.

Xu, Y., Li, Z., and Tan, K.S. (2008). Optimal investment with noise trading risk. Journal of Systems Science and Complexity 21, Iss. 4, 519-526.

Zhang, L., and Li, Z. (2012). Multi-period mean-variance portfolio selection with uncertain time horizon when returns are serially correlated. Mathematical Problems in Engineering Vol. 2012, Article ID 216891, 17 pages. 


\section{Appendix: proofs}

In this Appendix, we provide the proof of Theorem 3.1 accordingly to the outline given above.

\section{A.1 Discrete Itô formula.}

The Discrete Itô formula which we use in this paper is similar to the formula first introduced by Appleby, Berkolaiko and Rodkina (2009) for the proof of stability results for scalar stochastic difference equations. The main purpose of this formula is to mimic the classical Itô formula for continues processes when we deal with the discrete process described by the equation with small parameter $h$, similar (2.1). Berkolaiko et al (2012) demonstrates the use of a discrete Itô formula in the context of stochastic numerical analysis. Theorem A.1 below deals with the case which is slightly different than the one considered in Appleby, Berkolaiko and Rodkina (2009) and Berkolaiko et al (2012). Theorem A.1 can also be obtained as a partial case of Lemma 5.1 from Rodkina and Dokuchaev (2013), where the Itô formula was proved for the diagonal system of stochastic difference equations. However it is much easier to give a sketch of the proof here than to adapt Lemma 5.1 for (2.1).

Theorem A.1. Let Assumptions 2.1, 2.2, and condition (2.7) hold. Consider $\phi: \mathbf{R} \rightarrow \mathbf{R}$ such that there exists $\delta \in(0,1)$ and $\phi_{\delta}: \mathbf{R} \rightarrow \mathbf{R}$ saisfying

(i) $\phi_{\delta}$ has a bounded third derivative on $\mathbf{R}$,

(ii) $\phi_{\delta}(s)=\phi(s)$ for $s \notin(-\delta, \delta)$,

(iii) $\left|\phi_{\delta}(s)-\phi(s)\right|<K$ for some $K>0$ and all $s \in(-\delta, \delta)$.

Then there exists $h_{0}$ such that, for all $h \leq h_{0}, N \geq \frac{T}{h_{0}}$, and $n=0,1, \ldots, N-1$

$$
\mathbf{E}\left(\phi\left(1+h u_{n} a_{n}+\sqrt{h} u_{n} b_{n} \xi_{n+1}\right)\right)=\phi(1)+h \phi^{\prime}(1) u_{n} a_{n}+h \frac{\phi^{\prime \prime}(1)}{2} u_{n}^{2} b_{n}^{2}+o(h),
$$

where

$$
|o(h)| \leq h^{3 / 2} \hat{K} u_{n}^{2} b_{n}^{2}
$$

and $\hat{K}>0$ does not depend on $N$.

Proof. Fix $n=0,1, \ldots, N-1$ and define

$$
\zeta_{n+1}:=1+h u_{n} a_{n}+\sqrt{h} u_{n} b_{n} \xi_{n+1}, \quad \nu_{n+1}:=h u_{n} a_{n}+\sqrt{h} u_{n} b_{n} \xi_{n+1},
$$

and, for all $v \in \mathbb{R}$,

$$
\eta(v):=1+h u_{n} a_{n}+\sqrt{h} u_{n} b_{n} v .
$$


Expand $\phi_{\delta}\left(\zeta_{n+1}\right)$ by Taylor's formula and apply mathematical expectation

$$
\mathbf{E} \phi_{\delta}\left(\zeta_{n+1}\right)=\phi_{\delta}(1)+\phi_{\delta}^{\prime}(1) \mathbf{E} \nu_{n+1}+\frac{\phi_{\delta}^{\prime \prime}(1)}{2} \mathbf{E} \nu_{n+1}^{2}+\mathbf{E}\left[\frac{\phi_{\delta}^{\prime \prime \prime}(\theta)}{6} \nu_{n+1}^{3}\right]
$$

where $\theta$ is situated between 1 and $1+h u_{n} a_{n}+\sqrt{h} u_{n} b_{n} \xi_{n+1}$. Applying (2.5) we arrive at the estimate

$$
\begin{aligned}
\left|\mathbf{E}\left[\frac{\phi_{\delta}^{\prime \prime \prime}(\theta)}{6} \nu_{n+1}^{3}\right]\right| \leq & K_{1} \mathbf{E}\left|h u_{n} a_{n}+\sqrt{h} u_{n} b_{n} \xi_{n+1}\right|^{3} \\
& \leq K_{2}\left|u_{n}\right|^{3} h^{3 / 2}\left[h a_{n}^{3}+3 a_{n} b_{n}^{2}\right] \leq K_{3} u_{n}^{2} b_{n}^{2} h^{3 / 2},
\end{aligned}
$$

where $K_{i}, i=1,2,3$, does not depend on $n$. Note also that

$$
\phi_{\delta}(1)=\phi(1), \quad \phi_{\delta}^{\prime}(1)=\phi^{\prime}(1), \quad \phi_{\delta}^{\prime \prime}(1)=\phi^{\prime \prime}(1) .
$$

So the only thing which needs to be done is to estimate

$$
\Delta_{2}:=\mathbf{E}\left|\phi\left(\zeta_{n+1}\right)-\phi_{\delta}\left(\zeta_{n+1}\right)\right|=\frac{1}{\sqrt{2 \pi}} \int_{\mid(\eta(v) \mid \leq \delta}\left|\phi(\eta(v))-\phi_{\delta}(\eta(v))\right| e^{-v^{2} / 2} d v .
$$

Change the variables by

$$
s=1+h u_{n} a_{n}+\sqrt{h} u_{n} b_{n} v, \quad v=\frac{s-1-h u_{n} a_{n}}{\sqrt{h} u_{n} b_{n}}, \quad d v=\frac{d s}{\sqrt{h} u_{n} b_{n}} .
$$

Assume that $\delta$ and $h_{0}>0$ are small enough and $|s| \leq \delta, h \leq h_{0}$. Then, for $u_{n} b_{n}>0$ we have

$$
v=\frac{s-1-h u_{n} a_{n}}{\sqrt{h} u_{n} b_{n}} \leq \frac{\delta-1-h u_{n} a_{n}}{\sqrt{h} u_{n} b_{n}} \leq-\frac{1}{2 \sqrt{h} u_{n} b_{n}} \leq-\frac{1}{2 \sqrt{h} \hat{u} \hat{b}},
$$

while $u_{n} b_{n}<0$ we have

$$
v=\frac{1-s-h\left|u_{n} a_{n}\right|}{\sqrt{h}\left|u_{n} b_{n}\right|} \geq \frac{1-\delta-h\left|u_{n} a_{n}\right|}{\sqrt{h}\left|u_{n} b_{n}\right|} \geq \frac{1}{2 \sqrt{h}\left|u_{n} b_{n}\right|} \geq \frac{1}{2 \sqrt{h} \hat{u} \hat{b}} .
$$

So

$$
|v| \geq \frac{1}{2 \sqrt{h}\left|u_{n} b_{n}\right|}
$$

which implies that

$$
e^{-v^{2} / 2} \leq K_{4} v^{-4} \leq K_{5} h^{2} u_{n}^{4} b_{n}^{4} .
$$

Note that $h_{0}>0$ chosen here does not depend on $n$, but only on bounds for $a, b, u$, i.e. on $\hat{a}, \hat{b}, \hat{u}, \underline{a}, \underline{b}, \underline{u}>0$ (see $(2.5),(2.7))$.

This gives us

$$
\begin{aligned}
\Delta_{2}= & \frac{1}{\sqrt{2 \pi}} \int_{\mid(\eta(v) \mid \leq \delta}\left|\phi(\eta(v))-\phi_{\delta}(\eta(v))\right| e^{-v^{2} / 2} d v \\
& \leq \frac{K_{5} h^{2} u_{n}^{4} b_{n}^{4}}{\sqrt{2 \pi}} \int_{\mid(\eta(v) \mid \leq \delta}\left|\phi(\eta(v))-\phi_{\delta}(\eta(v))\right| d v \\
& =\frac{K_{5} h^{2} u_{n}^{4} b_{n}^{4}}{\sqrt{2 \pi} \sqrt{h} u_{n} b_{n}} \int_{|s| \leq \delta}\left|\phi(s)-\phi_{\delta}(s)\right| d s \leq K_{6} h^{3 / 2} u_{n}^{2} b_{n}^{2}
\end{aligned}
$$


where $K_{6}$ does not depend on $n$, which completes the proof.

Corollary 1.1. For $\phi$, defined by (3.7), formula (A.1) takes the form

$$
\begin{aligned}
& \mathbf{E}\left(\phi\left(1+h u_{n} a_{n}+\sqrt{h} u_{n} b_{n} \xi_{n+1}\right)\right) \\
& =1+h \alpha u_{n} a_{n}+h \frac{\alpha(\alpha-1)}{2} u_{n}^{2} b_{n}^{2}+o(h),
\end{aligned}
$$

where

$$
|o(h)| \leq h^{3 / 2} \hat{K} u_{n}^{2} b_{n}^{2},
$$

and $\hat{K}>0$ does not depend on $n=0,1, \ldots, N-1$. Hence (A.2) can be written as

$$
\begin{aligned}
& \mathbf{E}\left(\phi\left(1+h u_{n} a_{n}+\sqrt{h} u_{n} b_{n} \xi_{n+1}\right)\right) \\
& =1+h \alpha u_{n} a_{n}+h \frac{\alpha(\alpha-1)}{2} u_{n}^{2} b_{n}^{2}\left[1+h^{1 / 2} O_{n}(1)\right],
\end{aligned}
$$

uwhere $\left|O_{n}(1)\right| \leq \hat{K}$ for all $n=0,1, \ldots, N-1, N>\frac{T}{h_{0}}$ and $h \leq h_{0}$.

\section{A.2 Positivity of $x_{n}$ with probability close to 1}

In this section we follow ideas from Appleby et al (2010), showing that even though a.s. positivity is impossible to achieve for solution of (2.1), positivity with arbitrarily high probability is observed as the number of mesh points $N$ grows large. Again, we are giving the sketch of the proof instead of adapting a result from Appleby et al (2010).

Let $x_{n}$ be a solution to (2.1) with a positive initial value $x_{0}>0$, a parameter $h=\frac{T}{N}$ and a strategy $u$. Define

$$
\Omega_{N}:=\mathbb{P}\left\{\omega \in \Omega: x_{n}(\omega)>0, \quad n=1, \ldots, N\right\} .
$$

Lemma A.1. Let Assumptions 2.1, 2.2 and condition (2.7) hold. Let $\Omega_{N}$ be defined as in (A.4). Then, for each $\gamma \in(0,1)$, we can find $N(\gamma)$ such that for all $N \geq N(\gamma)$

$$
\mathbb{P}\left[\Omega_{N}\right] \geq 1-\gamma
$$

Proof. Note that $x_{n}$ is $\mathcal{F}_{n}$-measurable and is independent of $\xi_{n+1}$. Let $u_{n} b_{n}>0$. Then, for $n=0,1, \ldots, N-1$, we have

$$
\begin{aligned}
& \mathbb{P}\left\{x_{n+1}>0 \mid x_{n}>0\right\}=\mathbb{P}\left\{x_{n}\left(1+h u_{n} a_{n}+\sqrt{h} u_{n} b_{n} \xi_{n+1}\right)>0 \mid x_{n}>0\right\} \\
& =\mathbb{P}\left\{1+h u_{n} a_{n}+\sqrt{h} u_{n} b_{n} \xi_{n+1}>0 \mid x_{n}>0\right\}=\mathbb{P}\left\{\xi_{n+1}>-\frac{1+h u_{n} a_{n}}{\sqrt{h} u_{n} b_{n}} \mid x_{n}>0\right\} \\
& =1-\Phi\left(-\frac{1+h u_{n} a_{n}}{\sqrt{h} u_{n} b_{n}}\right)=\Phi\left(\frac{1+h u_{n} a_{n}}{\sqrt{h} u_{n} b_{n}}\right),
\end{aligned}
$$


where $\Phi$ is a normal probability distribution function.

If $u_{n} b_{n}<0$ we consider $\bar{\xi}_{n+1}=-\xi_{n+1}$ and note that $\bar{\xi}_{n+1}$ is also standard normal variable. So calculations (A.5) holds true in this case again.

Applying (A.5), Mill's estimate (see Karatzas and Shreve (1991))

$$
\frac{x}{\left(1+x^{2}\right) \sqrt{2 \pi}} e^{-x^{2} / 2} \leq 1-\Phi(x) \leq \frac{1}{x \sqrt{2 \pi}} e^{-x^{2} / 2}, \quad x>0,
$$

and the inequality

$$
\frac{1+h u_{n} a_{n}}{\sqrt{h} u_{n} b_{n}} \geq \frac{1}{2 \sqrt{h} \hat{u} \hat{b}}
$$

we conclude that for some $h_{1}>0$ and all $h<h_{1}$, we have

$$
\begin{aligned}
& \mathbb{P}\left\{x_{n+1}>0 \mid x_{n}>0\right\} \geq \Phi\left(\frac{1+h u_{n} a_{n}}{\sqrt{h} u_{n} b_{n}}\right) \geq 1-\frac{1}{\sqrt{2 \pi}} \frac{e^{-\frac{1}{2}\left(\frac{1+h u_{n} a_{n}}{\sqrt{h} u_{n} b_{n}}\right)^{2}}}{\frac{1+h u_{n} a_{n}}{\sqrt{h} u_{n} b_{n}}} \\
& \geq 1-K_{1} \frac{\left(\frac{1}{2 \sqrt{h} \hat{u} \hat{b}}\right)^{-3}}{\frac{1}{2 \sqrt{h} \hat{u} \hat{b}}}=1-K_{1}(2 \sqrt{h} \hat{u} \hat{b})^{4}=1-K_{2} h^{2},
\end{aligned}
$$

where $K_{1}, K_{2}>0$ do not depend on $n$. Then,

$$
\begin{aligned}
\mathbb{P}\left[\Omega_{N}\right] & :=\prod_{n=0,1, \ldots N-1} \mathbb{P}\left\{x_{n+1}>0 \mid x_{n}>0\right\} \geq \prod_{n=0,1, \ldots N-1}\left(1-K_{2} h^{2}\right) \\
& =\left(1-K_{2} h^{2}\right)^{N}=\left(1-\frac{K_{2} T^{2}}{N^{2}}\right)^{N} .
\end{aligned}
$$

Fix now $\gamma \in(0,1)$ and find $N(\gamma)$ such that for all $N \geq N(\gamma)$

$$
1-\left(1-\frac{K_{2} T^{2}}{N^{2}}\right)^{N}<\gamma
$$

This implies that for all $N \geq N(\gamma)$

$$
\mathbb{P}\left[\Omega_{N}\right] \geq 1-\gamma
$$

which completes the proof.

\section{A.3 Estimation of maximum $\mathbf{E} \phi\left(x_{N}\right)$}

Let $h_{0}, O_{n}(1)$ and $\hat{K}$ be from Corollary 1.1. So formula (A.3) holds and $\left|O_{n}(1)\right|<\hat{K}$ for all $n=1, \ldots, N-1, N>N_{0}=\frac{T}{h_{0}}$. In addition we assume that $h_{0}$ is so small that for $h \leq h_{0}$, $n=1, \ldots, N-1, N>N_{0}$,

$$
1-h^{1 / 2} \hat{K}>0, \quad 1+h \alpha a_{n} u_{n}+h \frac{\alpha(\alpha-1)}{2} u_{n}^{2} b_{n}^{2}\left[1-\hat{K} h^{1 / 2}\right]>0 .
$$


Define, for $h \leq h_{0}, N>N_{0}$ and for any admissible strategy $u$,

$$
\begin{aligned}
G(u):=\mathbf{E} \phi\left(x_{N}\right) & =\phi\left(x_{0}\right) \prod_{n=0}^{N-1} \mathbf{E}\left(\phi\left(1+h u_{n} a_{n}+\sqrt{h} u_{n} b_{n} \xi_{n+1}\right)\right) \\
& =x_{0}^{\alpha} \prod_{n=0}^{N-1}\left[1+\alpha h u_{n} a_{n}+h \frac{\alpha(\alpha-1)}{2} u_{n}^{2} b_{n}^{2}\left[1+h^{1 / 2} O_{n}(1)\right]\right],
\end{aligned}
$$

and

$$
\mathcal{G}(u):=x_{0}^{\alpha} \prod_{n=0}^{N-1}\left[1+\alpha h u_{n} a_{n}+h \frac{\alpha(\alpha-1)}{2} u_{n}^{2} b_{n}^{2}\right]
$$

\section{Calculation of strategy to maximize $\mathcal{G}(u)$}

Lemma A.2. Let Assumptions 2.1 and condition 2.7 hold. Let $\mathcal{G}$ be defined as in (A.8). Then the strategy $u^{*}$, defined by (3.6), maximizes $\mathcal{G}(u)$.

Proof. To find the maximum of $\mathcal{G}$ we calculate its partial derivatives. We have

$$
\frac{\partial \mathcal{G}}{\partial u_{k}}=x_{0}^{2} \alpha h\left(a_{k}+(\alpha-1) u_{k} b_{k}^{2}\right) \prod_{n=0, n \neq k}^{N-1}\left[1+h \alpha a_{n} u_{n}+h \frac{\alpha(\alpha-1)}{2} u_{n}^{2} b_{n}^{2}\right] .
$$

By (A.6), solving the system

$$
\frac{\partial \mathcal{G}}{\partial u_{k}}=0, \quad k=0,1, \ldots, N-1
$$

is equivalent to solving the system

$$
a_{k}+(\alpha-1) u_{k} b_{k}^{2}=0, \quad k=0,1, \ldots, N-1
$$

Solution $u^{*}$ to (A.9) is given by (3.6). To show that $u^{*}$ is a point of maximum for the function $\mathcal{G}$, we find second partial derivatives of $\mathcal{G}$ at $u^{*}$. We have, for $k=0, \ldots, N-1$,

$$
\frac{\partial^{2} \mathcal{G}}{\partial u_{k}^{2}}=x_{0}^{2} \alpha h(\alpha-1) b_{n}^{2} \prod_{n=0, n \neq k}^{N-1}\left[1+\alpha h a_{n} u_{n}+h \frac{\alpha(\alpha-1)}{2} u_{n}^{2} b_{n}^{2}\right],
$$

and, for $k \neq j$,

$$
\begin{aligned}
& \frac{\partial^{2} \mathcal{G}}{\partial u_{k} \partial u_{j}}=x_{0}^{2} \alpha^{2} h^{2}\left(a_{k}+(\alpha-1)\right.\left.u_{k} b_{k}^{2}\right)\left(a_{j}+(\alpha-1) u_{j} b_{j}^{2}\right) \\
& \times \prod_{n=0, n \neq k, j}^{N-1}\left[1+\alpha h a_{n} u_{n}+h \frac{\alpha(\alpha-1)}{2} u_{n}^{2} b_{n}^{2}\right] .
\end{aligned}
$$

Let $y=\left(y_{0}, \ldots, y_{N-1}\right)$. Consider the following quadratic form

$$
Q(y)=\left.\sum_{k, j=0}^{N-1} \frac{\partial^{2} \mathcal{G}}{\partial u_{k} \partial u_{j}}\right|_{u=u^{*}} y_{k} y_{j}
$$


Since

$$
\left(a_{k}+(\alpha-1) u_{k}^{*} b_{k}^{2}\right)\left(a_{j}+(\alpha-1) u_{j}^{*} b_{j}^{2}\right)=0,
$$

we have, for $k \neq j$,

$$
\left.\frac{\partial^{2} \mathcal{G}}{\partial u_{k} \partial u_{j}}\right|_{u=u^{*}}=0
$$

and (A.10) takes the form

$$
Q(y)=\left.\sum_{k=0}^{N-1} \frac{\partial^{2} G}{\partial u_{k}^{2}}\right|_{u=u^{*}} y_{k}^{2}=x_{0}^{2} \alpha h(\alpha-1) \sum_{k}^{N} b_{k}^{2} \prod_{n=0, n \neq k}^{N-1}\left[1+\frac{\alpha h a_{n}^{2}}{2(1-\alpha) b_{n}^{2}}\right] y_{k}^{2} .
$$

Since $\alpha-1<0$, but

$$
\alpha h>0, \quad b_{k}^{2}>0, \quad 1+\frac{\alpha h a_{n}^{2}}{2(1-\alpha) b_{n}^{2}}>0,
$$

the quadratic form $Q(y)$ is negatively defined, which proves that $u^{*}$ given by (3.6) is a point of maximum for $\mathcal{G}$.

\section{Estimation of the difference $G(u)-\mathcal{G}(u)$}

Lemma A.3. Let Assumptions 2.1, 2.2 and condition (2.7) hold. Let $\mathcal{G}(u)$ and $G(u)$ be defined as in (A.8) and (A.7), respectively. Then, for each $\varepsilon>0$ there exists $N(\varepsilon) \in \mathbf{N}$ such that for all $N>N(\varepsilon), h \leq \frac{T}{N(\varepsilon)}$, we have

$$
|G(u)-\mathcal{G}(u)| \leq \varepsilon
$$

Proof. Denote

$$
\nu_{n}:=1+\alpha h u_{n} a_{n}+h \frac{\alpha(\alpha-1)}{2} u_{n}^{2} b_{n}^{2}, \quad \eta_{n}:=\frac{\alpha(\alpha-1)}{2} \frac{u_{n}^{2} b_{n}^{2}}{\nu_{n}} .
$$

Let $h_{0}$ and $\hat{K}$ be as in Corollary 1.1. Assume in addition that $h_{0}$ is so small that

$$
1+\alpha h u_{n} a_{n}+h \frac{\alpha(\alpha-1)}{2} u_{n}^{2} b_{n}^{2}\left[1-h^{1 / 2} \hat{K}\right]>\frac{1}{2} .
$$

Based on Assumption 2.1, inequality (2.7) and (A.13), we conclude that there exist constants $\hat{K}_{1}>0$ and $\hat{K}_{2}>0$ which do not depend on $N\left(h_{0}\right)$ such that, for all $n=1,2, \ldots, N-1$,

$$
\hat{K} \eta_{n} \leq \hat{K}_{1}, \quad \hat{K} \alpha(\alpha-1) u_{n}^{2} b_{n}^{2} \leq \hat{K}_{2} .
$$

Note that $\left|O_{n}(1)\right| \leq \hat{K}$. Then, applying (A.13) and (A.14) we have, for all $n=1,2, \ldots, N-1$,

$$
\begin{aligned}
& 1+\alpha h u_{n} a_{n}+h \frac{\alpha(\alpha-1)}{2} u_{n}^{2} b_{n}^{2}\left[1+h^{1 / 2} O_{n}(1)\right] \leq \nu_{n}+h^{3 / 2} \hat{K} \frac{\alpha(\alpha-1)}{2} u_{n}^{2} b_{n}^{2} \\
& =\nu_{n}\left[1+h^{3 / 2} \hat{K} \eta_{n}\right] \leq \nu_{n} e^{h^{3 / 2} \hat{K} \eta_{n}} \leq \nu_{n} e^{h^{3 / 2} \hat{K}_{1}},
\end{aligned}
$$


and

$$
\begin{aligned}
& 1+\alpha h u_{n} a_{n}+h \frac{\alpha(\alpha-1)}{2} u_{n}^{2} b_{n}^{2}\left[1+h^{1 / 2} O_{n}(1)\right] \geq \nu_{n}-h^{3 / 2} \hat{K} \frac{\alpha(\alpha-1)}{2} u_{n}^{2} b_{n}^{2} \\
& =\nu_{n}\left[\frac{\nu_{n}-h^{3 / 2} \hat{K} \frac{\alpha(\alpha-1)}{2} u_{n}^{2} b_{n}^{2}}{\nu_{n}}\right]=\nu_{n}\left[\frac{\nu_{n}}{\nu_{n}-h^{3 / 2} \hat{K}^{\frac{\alpha(\alpha-1)}{2}} u_{n}^{2} b_{n}^{2}}\right]^{-1} \\
& =\nu_{n}\left[1+\frac{h^{3 / 2} \hat{K}^{\frac{\alpha(\alpha-1)}{2}} u_{n}^{2} b_{n}^{2}}{\nu_{n}-h^{3 / 2} \hat{K}^{\frac{\alpha(\alpha-1)}{2}} u_{n}^{2} b_{n}^{2}}\right]^{-1} \geq \nu_{n} \exp \left\{-\frac{h^{3 / 2} \hat{K}^{\frac{\alpha(\alpha-1)}{2}} u_{n}^{2} b_{n}^{2}}{\nu_{n}-h^{3 / 2} \hat{K}^{\frac{\alpha(\alpha-1)}{2}} u_{n}^{2} b_{n}^{2}}\right\} \\
& \geq \nu_{n} \exp \left\{-h^{3 / 2} \hat{K} \alpha(\alpha-1) u_{n}^{2} b_{n}^{2}\right\} \geq \nu_{n} e^{-h^{3 / 2} \hat{K}_{2} .}
\end{aligned}
$$

Note that

$$
\mathcal{G}(u)=x_{0}^{\alpha} \prod_{n=0}^{N-1} \nu_{n}, \quad h^{3 / 2} N=T h^{1 / 2}, \quad h N=T .
$$

Applying (A.16), (A.15) and (A.17) we arrive at

$$
\mathcal{G}(u) e^{-h^{3 / 2} \hat{K}_{2} N} \leq G(u) \leq \mathcal{G}(u) e^{h^{3 / 2} \hat{K}_{1} N},
$$

or

$$
\mathcal{G}(u) e^{-h^{1 / 2} \hat{K}_{2} T} \leq G(u) \leq \mathcal{G}(u) e^{h^{1 / 2} \hat{K}_{1} T}
$$

which implies that

$$
\mathcal{G}(u)\left[e^{-h^{1 / 2} \hat{K}_{2} T}-1\right] \leq G(u)-\mathcal{G}(u) \leq \mathcal{G}(u)\left[e^{h^{1 / 2} \hat{K}_{1} T}-1\right] .
$$

Therefore,

$$
|G(u)-\mathcal{G}(u)| \leq G(u) \max \left\{1-e^{-h^{1 / 2} \hat{K}_{2} T}, e^{h^{1 / 2} \hat{K}_{1} T}-1\right\} .
$$

Now we estimate $G(u)$. By Assumption 2.1 and inequality (2.7) we have

$$
\begin{aligned}
\mathcal{G}(u)= & x_{0}^{\alpha} \prod_{n=0}^{N-1}\left[1+\alpha h u_{n} a_{n}-h \frac{\alpha(1-\alpha)}{2} u_{n}^{2} b_{n}^{2}\right] \\
& \leq x_{0}^{\alpha} \exp \left\{\alpha h \sum_{n=0}^{N-1}\left[u_{n} a_{n}-\frac{(1-\alpha)}{2} u_{n}^{2} b_{n}^{2}\right]\right\} \\
& \leq x_{0}^{\alpha} \exp \left\{\alpha h N\left[\hat{a} \hat{u}-\frac{(1-\alpha)}{2} \underline{u}^{2} \underline{b}_{n}^{2}\right]\right\} \\
& =x_{0}^{\alpha} \exp \left\{\alpha T\left[\hat{a} \hat{u}-\frac{(1-\alpha)}{2} \underline{u}^{2} \underline{b}_{n}^{2}\right]\right\}=x_{0}^{\alpha} C_{1},
\end{aligned}
$$


for some $C_{1}>0$, which does not depend on $N$ or $h$.

Now, fix $\varepsilon>0$ and find $N=N(\varepsilon)$ such that, for $h<\frac{T}{N(\varepsilon)}$,

$$
\max \left\{e^{h^{1 / 2} 2 \hat{K}_{2}}-1,1-e^{-h^{1 / 2} 2 \hat{K}_{1}}\right\}<\frac{\varepsilon}{C_{1}} .
$$

Then, for $N>N(\varepsilon)$, inequality (A.11) holds.

\section{A.4 Estimation of $\max \mathbf{E} U\left(x_{N}\right)$}

Estimate for $\mathbf{E}\left|x_{N}\right|^{2}$

From (3.8) we obtain, for $n=1,2, \ldots, N$ :

$$
\begin{aligned}
& \mathbf{E}\left|x_{n}\right|^{2}=\left|x_{0}\right|^{2} \prod_{i=1}^{n-1} \mathbf{E}\left|1+h u_{i} a_{i}+\sqrt{h} u_{i} b_{i} \xi_{i+1}\right|^{2} \\
= & x_{0}^{2} \prod_{i=1}^{n-1} \mathbf{E}\left[1+h\left(2 u_{i} a_{i}+h u_{i}^{2} a_{i}^{2}+u_{i}^{2} b_{i}^{2}\right)+2 \sqrt{h}\left(1+h u_{i} a_{i}\right) u_{i} b_{i} \xi_{i+1}+h u_{i}^{2} b_{i}^{2}\left(\xi_{i+1}^{2}-1\right)\right] \\
= & x_{0}^{2} \prod_{i=1}^{n-1}\left[1+h\left(2 u_{i} a_{i}+h u_{i}^{2} a_{i}^{2}+u_{i}^{2} b_{i}^{2}\right)\right] \leq x_{0}^{2} \prod_{i=1}^{n-1}\left[1+h K_{3}\right] \leq\left|x_{0}\right|^{2}\left[1+h K_{3}\right]^{n},
\end{aligned}
$$

so

$$
\mathbf{E}\left|x_{N}\right|^{2} \leq x_{0}^{2}\left[1+h K_{3}\right]^{N}=\left|x_{0}\right|^{2} e^{N h K_{3}}=x_{0}^{2} e^{K_{3} T} .
$$

Estimate for $\max \mathbf{E} U\left(x_{N}\right)$.

Substituting the value $u^{*}$ from (3.6) into (A.8) we get

$$
\mathcal{G}\left(u^{*}\right)=x_{0}^{\alpha} \prod_{n=0}^{N-1}\left[1+\alpha h \frac{a_{n}^{2}}{2(1-\alpha) b_{n}^{2}}\right] .
$$

Lemma A.4. Let Assumptions 2.1, 2.2 and condition (2.7) hold. Let $x_{n}$ be a solution to (2.1) with a positive initial value $x_{0}>0$, a parameter $h=\frac{T}{N}$ and a strategy $u$. Let $\mathcal{G}\left(u^{*}\right)$ be defined as in (A.21) and $U$ be defined as in (2.6). Then, for each $\varepsilon>0$, there exists $N(\varepsilon) \in \mathbf{N}$ such that for all $N>N(\varepsilon), h \leq \frac{T}{N(\varepsilon)}$, we have

$$
\left|\sup _{u} \mathbf{E} U\left(x_{N}\right)-\mathcal{G}\left(u^{*}\right)\right| \leq \varepsilon
$$

Proof. Fix $\gamma \in(0,1)$ and find $N(\gamma)$ by Lemma A.1. Then, by definition (2.6) of $U$, for $\Omega_{N}$, defined by (A.4) with $N \geq N(\gamma)$, we have

$$
U\left(x_{N}(\omega)\right)=\phi\left(x_{N}(\omega)\right)=\left|x_{N}(\omega)\right|^{\alpha}, \quad \omega \in \Omega_{N},
$$


SO

$$
\mathbf{P}\left\{\omega: U\left(x_{N}(\omega)\right) \neq \phi\left(x_{N}(\omega)\right)\right\} \leq \mathbb{P}\left[\Omega \backslash \Omega_{N}\right] \leq \gamma .
$$

Further,

$$
\begin{aligned}
\mathbf{E} \mid \phi\left(x_{N}\right)-U & \left(x_{N}\right)\left|=\int_{\Omega}\right| \phi\left(x_{N}(\omega)\right)-U\left(x_{N}(\omega)\right) \mid d P(\omega) \\
& \leq \int_{\Omega \backslash \Omega_{N}}\left[\left|x_{N}(\omega)\right|^{\alpha}+L\left|x_{N}(\omega)\right|\right] d P(\omega) \\
& =\int_{\Omega \backslash \Omega_{N}}\left|x_{N}(\omega)\right|^{\alpha} d P(\omega)+L \int_{\Omega \backslash \Omega_{N}}\left|x_{N}(\omega)\right| d P(\omega) \\
& \leq\left(\int_{\Omega \backslash \Omega_{N}}\left|x_{N}(\omega)\right|^{2} d P(\omega)\right)^{\frac{\alpha}{2}} \times\left(\int_{\Omega \backslash \Omega_{N}} d P(\omega)\right)^{\frac{2-\alpha}{2}} \\
& +L\left(\int_{\Omega \backslash \Omega_{N}}\left|x_{N}(\omega)\right|^{2} d P(\omega)\right)^{\frac{1}{2}} \times\left(\int_{\Omega \backslash \Omega_{N}} d P(\omega)\right)^{\frac{1}{2}} \\
& \leq\left(\int_{\Omega}\left|x_{N}(\omega)\right|^{2} d P(\omega)\right)^{\frac{\alpha}{2}}\left(\mathbf{P}\left\{\Omega \backslash \Omega_{N}\right\}\right)^{\frac{2-\alpha}{2}}+ \\
& L\left(\int_{\Omega}\left|x_{N}(\omega)\right|^{2} d P(\omega)\right)^{\frac{1}{2}}\left(\mathbf{P}\left\{\Omega \backslash \Omega_{N}\right\}\right)^{\frac{1}{2}} \\
& \leq\left(\mathbf{E}\left|x_{N}\right|^{2}\right)^{\frac{\alpha}{2}} \gamma^{\frac{2-\alpha}{2}}+L\left(\mathbf{E}\left|x_{N}\right|^{2}\right)^{\frac{1}{2}} \gamma^{\frac{1}{2}}
\end{aligned}
$$

Since $1-\frac{\alpha}{2}>\frac{1}{2}$ and $\gamma \in(0,1)$ estimates (A.20) and (A.23) imply

$$
\mathbf{E}\left|\phi\left(x_{N}\right)-U\left(x_{N}\right)\right| \leq K_{4} \gamma^{\frac{1}{2}}
$$

where $K_{4}>0$ does not depend on $N$.

Then

$$
\left|\mathbf{E} U\left(x_{N}\right)-\mathbf{E} \phi\left(x_{N}\right)\right| \leq \mathbf{E}\left|\phi\left(x_{N}\right)-U\left(x_{N}\right)\right| \leq K_{4} \gamma^{\frac{1}{2}} .
$$

Now, fix $\varepsilon>0$, and choose

$$
\gamma<\left(\frac{\varepsilon}{2 K_{4}}\right)^{2} .
$$

By Lemma A.1, find $N(\gamma)$. By Lemma A.3, find $N(\varepsilon / 2) \geq N(\gamma)$ such that, for each admissible strategy $u, N \geq N(\varepsilon / 2)$ and $h \leq \frac{T}{N(\varepsilon / 2)}$ we have

$$
|G(u)-\mathcal{G}(u)| \leq \varepsilon / 2 .
$$

Recall that $\mathbf{E} \phi\left(x_{N}\right)=G(u)$ and $\sup _{u}[\mathcal{G}(u)]=\mathcal{G}\left(u^{*}\right)$. Then, by (A.24) and (A.25) we have, for 
$N \geq N(\varepsilon / 2)$ and $h \leq \frac{T}{N(\varepsilon / 2)}$

$$
\begin{aligned}
& \left|\sup _{u} \mathbf{E} U\left(x_{N}\right)-\mathcal{G}\left(u^{*}\right)\right|=\left|\sup _{u}\left[\mathbf{E} U\left(x_{N}\right)-\mathbf{E} \phi\left(x_{N}\right)+G(u)-\mathcal{G}(u)+\mathcal{G}(u)\right]-\mathcal{G}\left(u^{*}\right)\right| \\
& \leq\left|\sup _{u}\left[\mathbf{E} U\left(x_{N}\right)-\mathbf{E} \phi\left(x_{N}\right)\right]\right|+\left|\sup _{u}[G(u)-\mathcal{G}(u)]\right|+\left|\sup _{u}[\mathcal{G}(u)]-\mathcal{G}\left(u^{*}\right)\right| \\
& \leq K_{4} \gamma^{\frac{1}{2}}+\frac{\varepsilon}{2} \leq \varepsilon .
\end{aligned}
$$

Now we are able to complete the proof of Theorem 3.1. For small enough $h=\frac{T}{N}$, the strategy $u^{*}$ defined by (3.6) maximize $\mathbf{E} U\left(x_{N}\right)$ approximately, meaning that

$$
\sup _{u} \mathbf{E} U\left(x_{N}\right)=x_{0}^{2} \prod_{n=0}^{N-1}\left[1+\alpha h \frac{a_{n}^{2}}{2(1-\alpha) b_{n}^{2}}\right]+\rho(N),
$$

where $\rho(N) \rightarrow 0$ as $N \rightarrow \infty$. Then the proof of Theorem 3.1 follows. 